\title{
Cultural factors that influence obesity in children aged 3-12 years living in England and France
}

\author{
Y. Wake and S. Reeves \\ School of Human and Life Sciences, Roehampton University, Holybourne Avenue, London SW15 4JD, UK
}

Overweight and obesity in children is considered to be a world health problem. It is increasing in children of all ages and is of growing concern for governments across the globe. In Europe the incidence is high and current UK government data show that approximately $32 \%$ of 2-15 year olds are obese or overweight ${ }^{(1)}$. Whilst levels of obesity are lower in France, incidence rates of overweight and obese children were approximately $17 \%$ in $2006^{(2)}$. The present study aims to make a comparison of the incidence and cultural and lifestyle factors that affect obesity in children living in England and France.

Ninety-eight children from Monetier-les-Bains in France and 105 children from south west London, UK were invited to take part in the study. In total 146 children aged 3-12 years completed the study. Both groups had similar socio-economic backgrounds and were age matched. The anthropometric measurements taken were: weight; height; waist circumference. In addition, a questionnaire to assess background information, lifestyle, physical activity and food habits were given to the children as well as $7 \mathrm{~d}$ diet and physical activity diaries. $\chi^{2}$ and $t$ tests were used to analyse the data.

\begin{tabular}{|c|c|c|c|c|c|c|c|c|}
\hline & \multicolumn{4}{|c|}{ French } & \multicolumn{4}{|c|}{ English } \\
\hline & \multicolumn{2}{|c|}{ Girls } & \multicolumn{2}{|c|}{ Boys } & \multicolumn{2}{|c|}{ Girls } & \multicolumn{2}{|c|}{ Boys } \\
\hline & Mean & $\overline{\mathrm{SD}}$ & Mean & SD & $\overline{\text { Mean }}$ & $\mathrm{SD}$ & Mean & $\overline{\mathrm{SD}}$ \\
\hline Weight (kg) & $24.2 *$ & 60.7 & $24.0^{*}$ & 41.4 & 30.0 & 11.0 & 30.0 & 84.2 \\
\hline $\mathrm{BMI}\left(\mathrm{kg} / \mathrm{m}^{2}\right)$ & $15.4^{*}$ & 2.5 & $15.2 *$ & 1.65 & 17.9 & 7.3 & 17.6 & 4.4 \\
\hline Waist circumference $(\mathrm{cm})$ & $56^{*}$ & 0.6 & $55^{*}$ & 0.4 & 61 & 0.5 & 61 & 0.3 \\
\hline
\end{tabular}

Mean values were significantly different from those for English children: $* P<0.01$.

The results showed a significant difference $(P<0.01)$ between French and English children for weight, BMI, Z-scores and waist circumferences. Furthermore, $32 \%$ of the English group were classified as overweight or obese compared with none of the French children. There were significant differences $(P<0.01)$ in the levels of out-of-school physical activity undertaken by the French children $(2.30(\mathrm{SD} 0.73) \mathrm{h} / \mathrm{d})$ and that of the English children $(0.99$ (SD 0.66) h). The level of sedentary activity, television viewing and computer use was also significantly different (French children 1.49 (SD 0.54) h, English children 3.09 (SD 2.3) h; $P<0.01$ ). School dinners or a packed lunch were consumed by $95 \%$ of English children, whereas it was usual for French children to go home. In addition, $99 \%$ of the French children consumed homegrown produce compared with $1 \%$ of the English children.

Physical activity and consequently energy balance appear to be an important factor in the incidence of overweight and obesity between the French and English children. Factors such as the role of food in French culture, the emphasis on meals not snacks, the lack of fast-food outlets in the area of France studied, distances walked or cycled to and from school and choice of activities and hobbies undertaken are seen as the main positive influences on energy balance in the French children. These findings have highlighted areas worthy of intervention in the battle against obesity in the UK.

1. Foresight (2007) Tackling obesities: future choices—project report. http://www.foresight.gov.uk/OurWork/ActiveProjects/Obesity/KeyInfo/Index.asp

2. Summerbell C (2008) Are public health policies to tackle childhood obesity fair, safe and sensible? http://www.cwt.org.uk/pdfs/Ev2008_ carolynSummerbell.pdf 